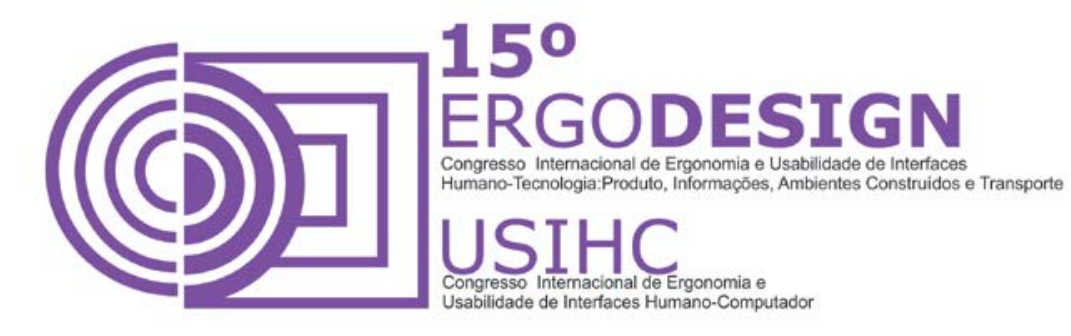

\title{
CARACTERIZAÇÃO E MODELAGEM DOS TIPOS DE ERRO HUMANO NA INTERAÇÃO COM SISTEMAS AUTOMATIZADOS
}

\section{CHARACTERIZATION AND MODELING OF HUMAN ERROR TYPES IN INTERACTION WITH AUTOMATED SYSTEMS}

\author{
(1) GONÇALVES, Rafael Cirino; \\ (2) QUARESMA, Manuela
}

(1) Laboratório de ergodesign e usabilidade de interfaces da PUC-Rio (LEUI), Graduando em Design

e-mail:rafaelcirinogoncalves@gmail.com

(2) Laboratório de ergodesign e usabilidade de interfaces da PUC-Rio (LEUI), Doutora em Design

e-mail:mquaresma@puc-rio.br

\begin{abstract}
RESUMO
Este artigo apresenta um estudo realizado sobre erros humanos no processo de interação com sistemas automatizados. O trabalho classifica e caracteriza os diferentes possíveis tipos de erro durante 0 processo de interação, apontando as principais causas e características. Os resultados da pesquisa foram obtidos a partir de um diagrama de afinidades e de uma modelagem dos erros de acordo com o esquema OODA LOOP.

Palavras-chave: Erro Humano; Automação; Segurança; Modelagem Cognitiva.

ABSTRACT

This article shows a study about human error during the human-automation interaction process. The paper classifies and characterizes different types of possible kinds of errors that may occur during the interaction process, pointing out the main causes and characteristics. The results were obtained through one affinity diagram and the modelling of the findings according to the OODA LOOP model.
\end{abstract}

Keywords: Human Error; Automation; Safety. Cognitive Modeling. 


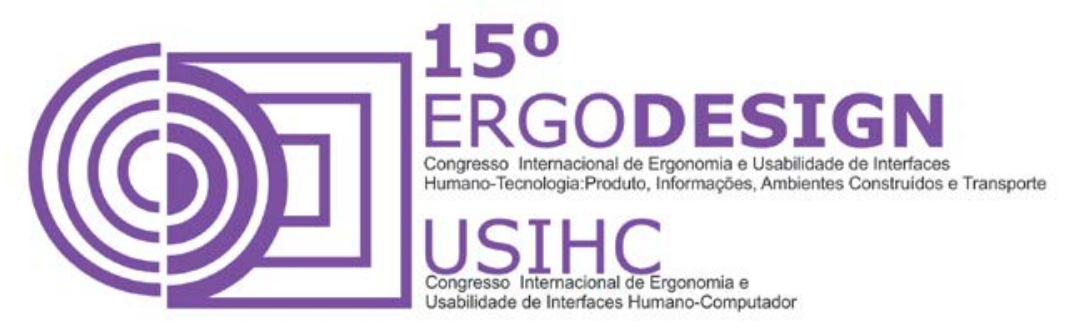

\section{INTRODUÇÃO}

Sistemas automatizados têm se tornado cada dia mais comuns na vida cotidiana. Tais aparelhos cumprem o objetivo de ajudar o homem nas suas atividades, de forma a reduzir a exigência de esforço para a sua execução. Apesar de sua eficiência, todas as ações de um sistema automatizado são baseadas em leituras numéricas de seus sensores, que são interpretadas e com isso presumem seu cenário de ação. Tal fato faz com que as ações destes mecanismos sempre estejam suscetíveis a erros, uma vez que computadores não são capazes de pensar de forma semântica, necessitando de uma abstração numérica que nem sempre corresponde à realidade.

Tal faliabilidade de sistemas automatizados pode comprometer a interação de diferentes maneiras, afetando diversos estágios da interação Humano-Máquina consequentemente levando a uma série de tipos de erro. Este estudo pretende analisar e caracterizar os diferentes tipos de erro humano durante a sua interação com sistemas automatizados, encontrando suas causas e principais aspectos. Para se chegar a tais resultados utilizou-se um diagrama de afinidades classificando os tipos de erro a partir de uma extensa revisão bibliográfica. Posteriormente, os achados foram modelados dentro do diagrama OODA LOOP (Thomas, 2001 apud Gikkas, 20013), para que pudessem ser melhor analisados.

\section{INTERAÇÃO HUMANO-AUTOMATIZAÇÃO:}

Automatização é um termo relativamente ainda em debate e que pode ter muitos significados, a depender do contexto em que se insere. Dentro deste estudo, entende-se automatização como a realização de tarefas e a atividades com a ação de máquinas, sem a interferência direta do humano no processo. De acordo com Parazuraman \& Sinderman (2011), automatização pode ser entendida em quatro vertentes: a) mecanização e integração de leituras de variáveis contextuais; b) processamento de dados e tomada de decisão por parte de computadores; c) ação mecânica que aplica força sobre determinado ambiente; d) ação informacional pela comunicação de informação processada para o usuário.

Em suma, o que é preciso entender sobre a automatização é que durante a interação com sistemas automatizados, o humano não mais é o ator primário na realização das tarefas a serem exercidas. Neste tipo de interação, funções são delegadas a máquinas, que por sua parte processam os dados relevantes para a situação, avaliam o contexto e agem de acordo com o que interpretam ser a melhor solução dentro de seus parâmetros. Com isso, entende-se que o operador da máquina só interage com os extremos iniciais e finais da operação, ou seja: o acionamento inicial e a recepção dos resultados, delegando a máquinas todas as etapas de cunho operacional.

Os estudos de base da ergonomia (Moraes \& Mont'Alvão, 2012; Chapanis, 1962; Proctor \& Vu, 2012; Fitts, 1959 apud. Salvendi, 2006) sempre tiveram como objeto principal as ações humanas utilizando máquinas como ferramentas para a realização de seus objetivos (estudos conhecidos como Análise da Tarefa), como pode ser visto no modelo descrito por Proctor \& Vu (2012) (figura 1): 

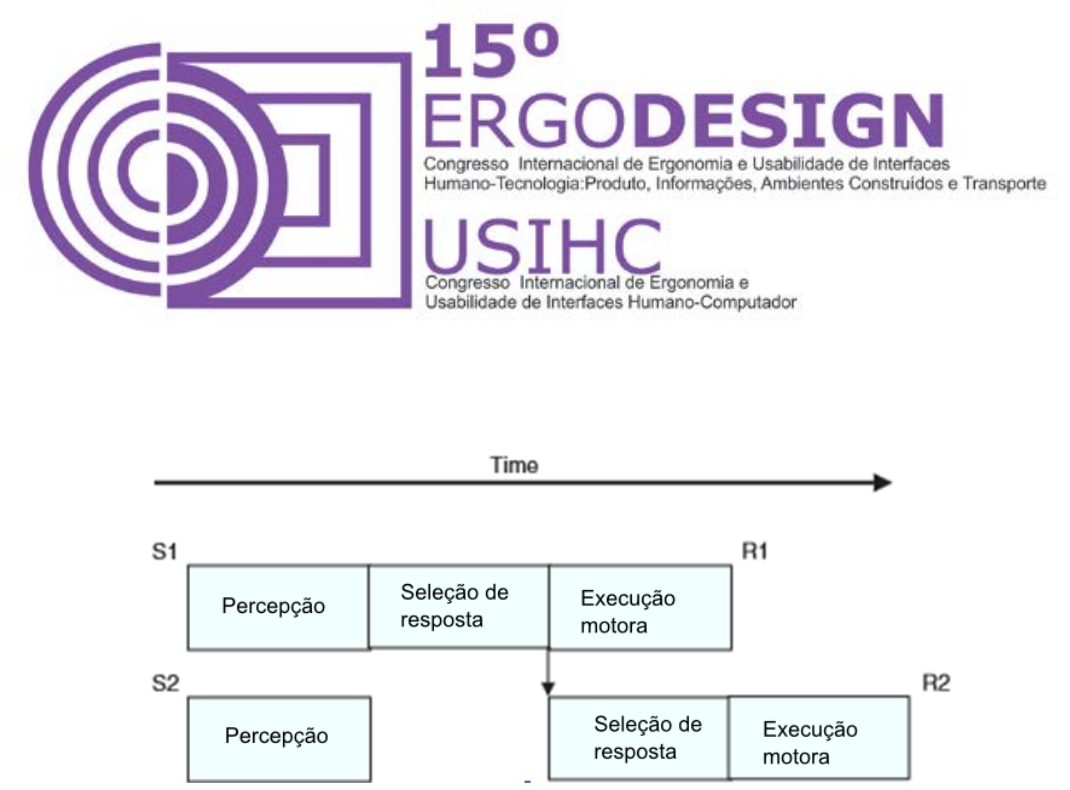

Figura 1 - Estrutura básica do Modelo de intração Humano-Máquina traduzido (Proctor \& Vu; 2012)

Porém, como afirmam Parazuraman \& Sinderman (2011), Degani (2003) e Norman (2006), a interação entre humanos e sistemas automatizados se dá de uma forma diferente, uma vez que o humano não mais é o agente principal para a realização da tarefa. Para melhor entender este tipo de interação, faz-se necessário observar a questão sobre outra perspectiva. Parasuraman \& Sinderman (2011) e Dekker (2005) definem um paradigma que ilustra a interação HumanoAutomatização, chamado como de paradigma de controle supervisório (Supervisory Control Paradigm). Tal paradigma pode ser entendido de forma que o humano, detentor do conhecimento geral dos objetivos da tarefa, coordena a máquina - detentora de capacidades operacionais para realizar atividades com precisão e destreza, para que a tarefa seja cumprida. Nesta visão, o humano assume um lugar de estrategista, cujo seu trabalho se resume a orientar as ações e observar sua execução de forma a garantir o bom funcionamento do processo.

O estado de controle supervisório pode ser subdividido em passos 5 (Parazuraman \& Sinderman, 2011; Dekker, 1997): a) planejamento da atividade offline; b) programar e orientar 0 sistema; c) monitoramento do sistema automatizado durante a execução da tarefa; d) interferir ou abortar a ação do sistema (caso haja algum erro de funcionamento); e) aprender com a experiência.

Pesquisadores da área de interação Humano-Automatização (Young, 2013; Degani, 2003; HSE, 2011; Parazuraman \& Sinderman, 2011) definem o processo de funcionamento de um sistema automatizado nas seguintes etapas: a) observação de uma variável, b) orientação desta variação de acordo com os limiares relevantes, decisão da ação necessária de acordo com o cenário observado, d) execução da ação.

Durante a interação Humano-Automatização os dois processos estão diretamente interligados, uma vez que cada uma das etapas de vigilância incide sobre cada um dos processos de funcionamento de sistemas automatizados. Um outro modelo que define a interação de forma mais detalhada é chamado de OODA LOOP (Observation, Orientation, Decision, Action) (Thomas, 2001 apud Gikkas, 2013) que leva em consideração não só os processos de interação, mas que elementos (subjetivos e concretos) interferem em cada uma das etapas.

OODA LOOP (Thomas, 2001 apud Gikkas 2013) - Figura 2- é um esquema com uma série de ações cognitivas e motoras requeridas de um operador/usuário para ele tomar decisões e executá-las durante a interação. Este esquema leva em consideração uma série de aspectos 


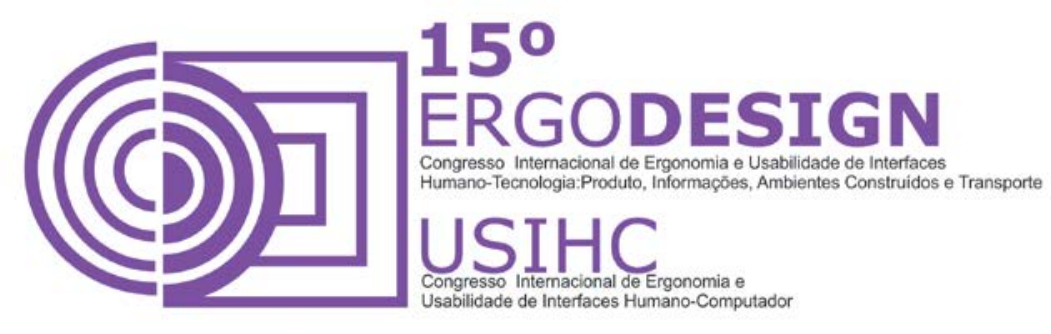

referentes ao usuário, como experiências prévias, aspectos culturais, informação dada ao usuário e de canais de atenção. O balanço e consideração de todas essas variáveis torna-se essencial durante a comunicação Humano-Automatização para que o usuário possa entender o que se passa durante a ação de um de um sistema automatizado e assim agir corretamente diante de cada situação.

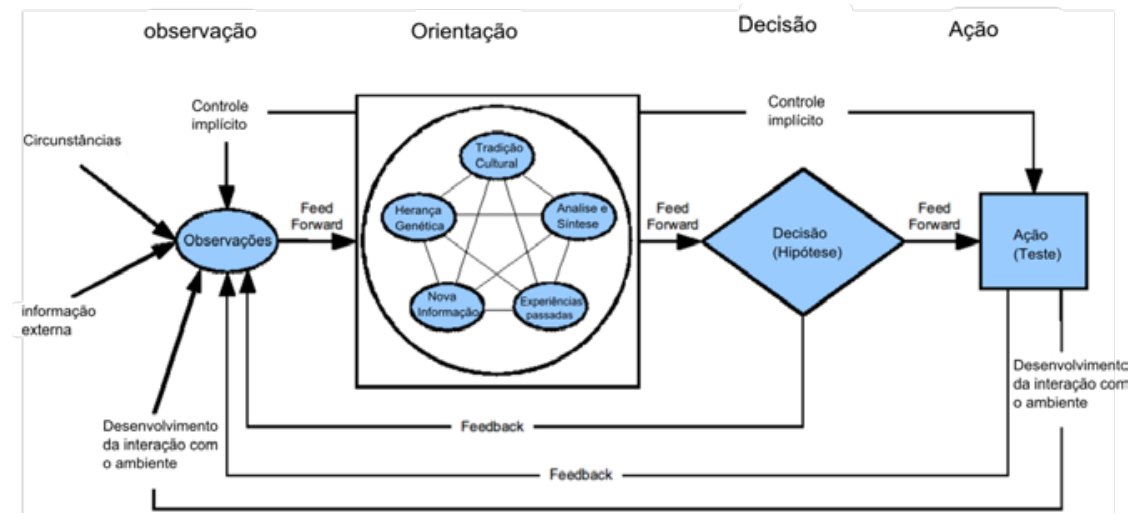

Figura 2 - Modelo OODA LOOP traduzido (Thomas, 2001 apud Gikkas 2013)

O esquema OODA LOOP também é aplicado para o funcionamento dos sistemas em si, levando em consideração as variáveis, cenários pré-determinados e leituras dos sensores para tomar as suas decisões. Durante a comunicação entre os usuários e o sistema, os modelos das duas partes estão diretamente interligados, uma vez que a falha de interpretação por parte dos sensores ou um feedback impreciso interfere diretamente nas ações do operador.

Ao observar o modelo acima (figura 2), pode-se perceber que fatores humanos subjetivos e culturais são de suma importância para o bom desempenho das tarefas envolvendo elementos automatizados - uma vez que a interpretação dos estados do sistema e do funcionamento do mesmo está em função das experiências prévias do usuário, assim como antecedentes culturais relacionados à automatização. Diversos autores (Parazuraman \& Sinderman, 2011; DEGANI, 2003; Thomas apud Gikkas, 2013) afirmam que um dos principais fatores que influenciam a interação Humano-Automatização são as experiências prévias dos usuários com este tipo de sistema.

\section{FALIABILIDADE DE SISTEMAS}

Apesar de sua eficiência, sistemas automáticos nem sempre são capazes de identificar com precisão o que realmente está ocorrendo quando a leitura dos dados é feita, levando a se tornar um fator de causa e não de prevenção de acidentes. Norman (2006) afirma que não existem objetos inteligentes, e sim responsivos, logo máquinas não tem como prever $100 \%$ dos casos presentes num sistema complexo. 


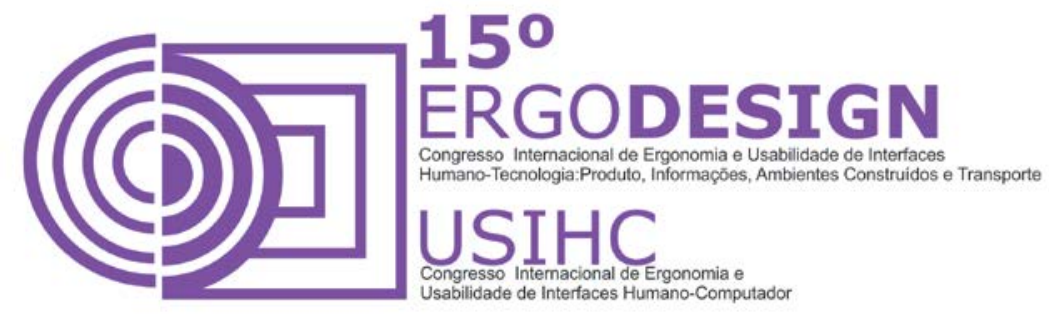

$\mathrm{Na}$ opinião de Norman (2006), a principal causa de nossa incapacidade de nos comunicarmos com as máquinas é o fato de não haver terreno comum de comunicação entre nós e elas.

O autor define o termo "terreno comum" como uma gama de conhecimentos comuns de compreensão necessária para o bom entendimento da mensagem entre dois interlocutores.

Norman (2006) afirma que: "pessoas e máquinas habitam universos diferentes, um de regras logicamente proscritas que governam a interação, o outro de ações complexas, dependentes do contexto, onde a mesma aparente condição dará origem a ações diferentes por que as circunstâncias são diferentes". Como agravante, existem questões fundamentais de objetivos e ações em que máquinas e humanos são incapazes de concordar - uma vez que leituras numéricas de máquinas não são capazes de traduzir valores humanos e relativos, se prendendo ao que pode ser diretamente analisado por seus sensores.

Como dito anteriormente, o funcionamento de um sistema automatizado pode ser divido nas seguintes etapas:

- Observação de mudança de uma variável.

- Orientação desta variação de acordo com os limiares relevantes.

- Decisão da manobra necessária de acordo com o cenário observado.

- Execução da ação.

O que se pode observar deste esquema é que todas as ações feitas pelos sistemas automatizados estão subordinadas a uma ou mais variáveis numéricas que em certos valores são interpretadas como determinada situação, sem necessariamente considerar erros de leitura ou fatores outros que possam apontar os mesmos valores em determinado sensor.

Tal incapacidade de interpretar com completa precisão determinados cenários faz com que sistemas estejam sempre sujeitos a erros.

HSE (2003) e Norman (2006) afirmam que as interpretações feitas por sistemas automatizados decorrem de padrões pré-definidos - possíveis cenários, pré-programados, com suas condições específicas de ocorrência. Porém, este modelo de funcionamento só é $100 \%$ preciso quando se tem controle de todas as variáveis interferindo no sistema, de forma a conseguir prever todos os cenários possíveis (vide processos de automatização fabril). Porém sistemas complexos como o trânsito possuem um número infinito de cenários prováveis, sendo inviável com a tecnologia atual encaixá-los em um número finito de ações de um sistema.

\section{PROBLEMAS DE INTERAÇÃO:}

Como pode ser visto acima, devido as suas limitações conceituais, máquinas podem ser suscetíveis a falhas, tanto de operação quanto de interpretação. Assim como os sistemas em si podem falhar, especificidades de interação mal resolvidas também podem causar problemas durante a realização de tarefas. Ambos os fatores tem um impacto negativo direto na 


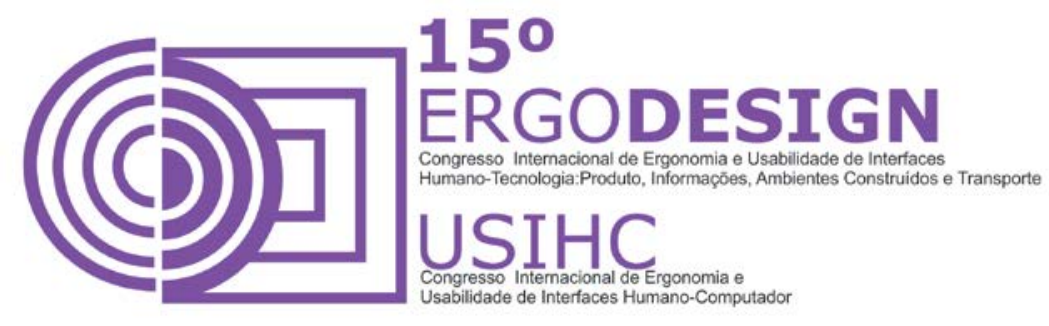

controlabilidade do sistema, comprometendo a interação em diferentes magnitudes a depender da gravidade do problema. Estes são os principais fatores causadores de erros durante a interação automatizada. Caso o usuário seja incapaz de retomar o controle do sistema, tais erros podem levar a acidentes (como pode ser visto na figura 3)

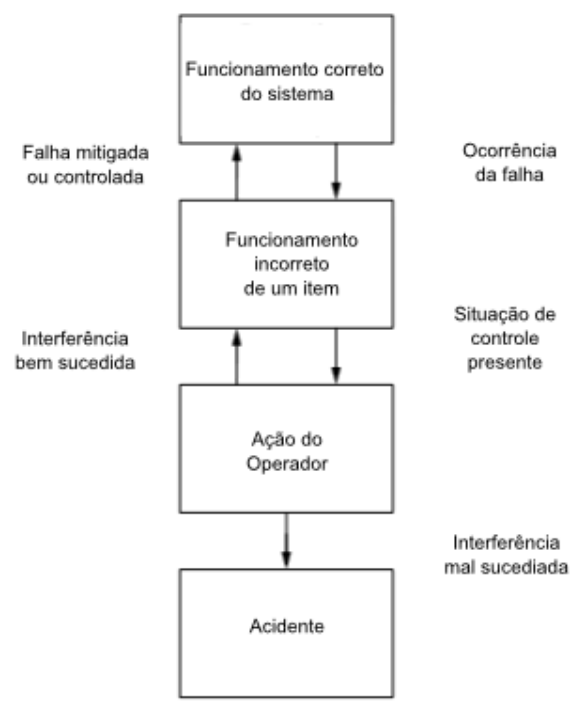

Figura 3 - Esquema de como riscos geram acidentes (ISO 26262)

Porém, não necessariamente falhas representam erros, nem mesmo erros necessariamente causam acidentes, todos estes problemas de interação podem ser remediados e mitigados, dando a possibilidade do usuário retomar o controle e corrigir a situação, reduzindo consequentemente a ocorrência de acidentes.

Harrys e Beringer (2007) apud Gikkas (2013) e HSE (2003) citam as possíveis causas de uma erros ou falhas em um sistema automatizado:

- Falta de treino dos operadores para utilizar o sistema;

- A falta de um modelo conceitual que auxilie o entendimento das operações realizadas automaticamente;

- Mal funcionamento devido a limitações dos sensores;

- Sistemas não instalados de acordo com as regras de segurança;

- Interpretações imprecisas podem oferecer um suporte não satisfatório para o usuário.

Pesquisadores como Young (2013), Parazuraman \& Rilley(2009), afirmam que problemas de interação Humano-Automatização comprometem 3 fatores fundamentais para a contolabilidade do sistema e aumentam o risco de acidentes, sendo eles: 


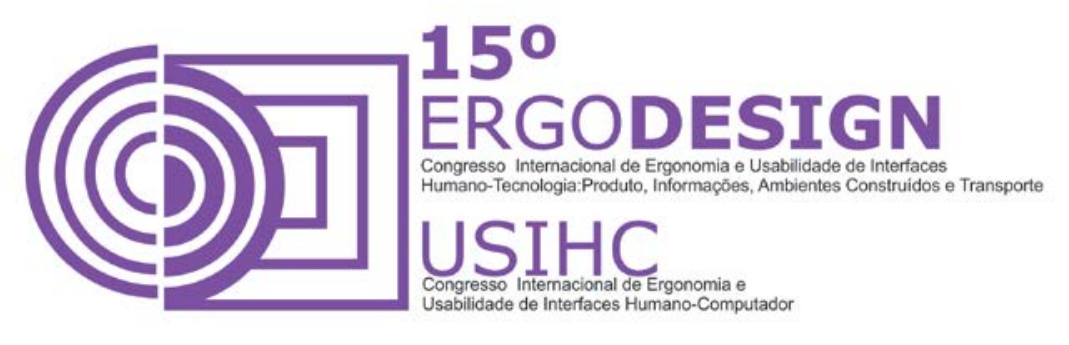

Confiança: Problemas de interação Humano-Automatização em relação a confiança se dão quando há uma previsão errada quanto ao funcionamento do sistema (Parazuraman 2009). Young (2013) afirma que os problemas podem ser causados tanto por excesso quanto por falta de confiança no sistema. Os problemas podem ser divididos e compostos em: a) auto confiança; b) confiança no sistema; c) confiabilidade do sistema. Esta confiança determina 0 nível de monitoramento do sistema automático por parte do operador - ora excessiva, comprometendo as outras tarefas, ora escassa, fazendo com que o usuário esteja despreparado para reagir. Young (2013) afirma que os principais problemas de confiança em máquinas envolvem a confiança do operador em relação a alarmes falsos e mudanças de comportamento.

Consciência da situação: Para Young (2013) e Kowalski (2013), consciência da situação pode ser definida como a real noção do modo de funcionamento, possibilitando uma pré-visualização dos futuros acontecimentos em diferentes cenários envolvendo a interação com sistemas automatizados. Para Young (2013), a falta de consciência da situação vem sido associada com uma supervisão passiva dos sistemas automatizados (out of the loop). Estar "out of the loop" pode ocasionar problemas de vigilância e de reação a emergências, ou recuperação de uma falha de automação. Young (2013) e Kowalsky (2013) afirmam que um dos principais problemas na automatização são os erros de modo (quando uma máquina muda de estado sem dar feedback para o usuário), fazendo com que ele tenha expectativas errôneas sobre as futuras ações do sistema e em situações críticas tenha que interpretar o funcionamento, aumentando o risco de acidentes. Grande parte dos erros humanos envolvendo automatização é causada pela falta de feedback do que o sistema está fazendo.

Carga de Trabalho: Como dito nos capítulos anteriores, a interferência de atividades complexas na atividade principal durante a interação com sistemas automatizados pode comprometer seu desempenho.

Apesar do aumento da carga cognitiva, Hughes \& Cole (1986) apud Gikkas (2013) afirmam que em alguns casos, uma redução excessiva da carga cognitiva do condutor pode ser prejudicial para a segurança da operação, visto que a mesma pode reduzir as chances de um usuário a reagir a uma súbita demanda de atenção.

Vale lembrar que todos os três problemas estão interligados entre si, de forma que a resolução de um dos fatores pode acarretar no outro, e vice e versa.

Kowalski (2013), Reason (1990) e outros definem erro humano como uma ação que produz resultados diferentes das expectativas. Para se observar o erro humano, é preciso considerar os processos psicológicos humanos e os fatores externos que influem na tarefa (Kowalski, 2013).

Sharit (2006) afirma que o erro humano é composto por três fatores: a) falibilidade humana limites de capacidades físicas humanas como fadiga e afins; b) contexto da tarefa - levando em consideração os objetivos, ferramentas e processos; c) barreiras - desafios, problemas de usabilidade, dificuldades motoras e afins. Como pode ser visto, o erro humano pode ser diretamente relacionado com os elementos que compõem a relação Humano-Automatização, 


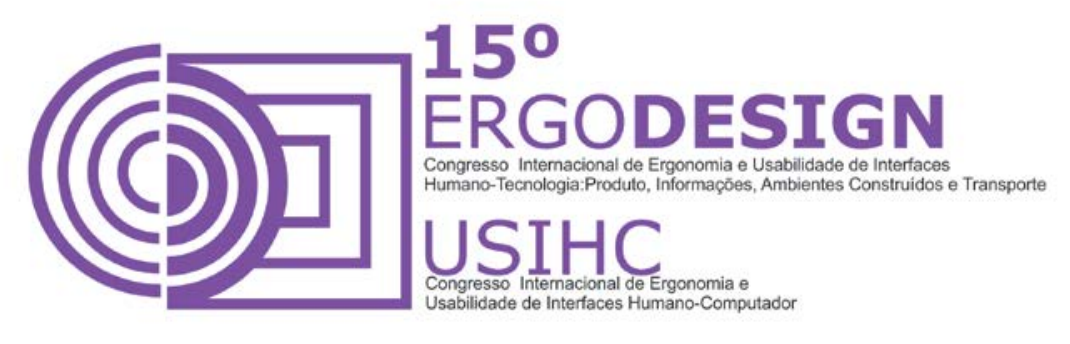

uma vez que o ruído em qualquer um dos processos de vigilância do sistema por parte do operador, assim como erros da máquina, comprometem a realização da tarefa.

Durante a interação Humano-Automatização, podem haver diversos tipos de erro, cada um deles relacionado aos processos descritos acima, tanto de supervisão por parte do operador, quanto de funcionamento do sistema automatizado, de forma que a falha na relação em qualquer uma das etapas pode ser prejudicial para a interação (Parazuraman \& Sinderman, 2011).

\section{METODOLOGIA}

Este estudo teve como objetivo classificar e compreender causas e características dos diferentes tipos de erro humano na interação Humano-Automatização. Para se alcançar os resultados desejados, optou-se pela seguinte metodologia:

- Extensa revisão bibliográfica dos principais autores na área de interação HumanoAutomatização.

- Consolidação dos tipos de erro recolhidos durante a revisão bibliográfica a partir de um diagrama de afinidades.

- Modelagem dos erros consolidados dentro do esquema OODA LOOP para possibilitar uma melhor análise das características do tipo de erro.

Para melhor entender as questões que envolvem as relações entre o humano e sistemas automatizados, foi feita uma extensa revisão bibliográfica com os principais autores da área (Reason, 1990; Dekker, 1997; Sharit, 2006; Parazuraman, 2011; Degani, 2003; Kletz, 2013). Tal revisão possibilitou um maior entendimento das principais questões que definem a taxonomia da interação Humano-Automatização. Com o entendimento do passo a passo deste tipo de tarefa, foi possível identificar uma série de definições sobre o tema e consequentemente, dentro deste conteúdo foi encontrada uma grande lista de tipos de erro humano na interação com sistemas automatizados.

Devido ao grande número de tipos de erro humano encontrados e uma ausência de padrão tanto de listagem quanto de nomenclatura, optou-se por se fazer uma consolidação e catalogação dos tipos de erro encontrados. O objetivo desta catalogação foi unificar os achados da revisão bibliográfica em uma única lista que abarcasse o conteúdo específico de cada um dos autores, unindo o que fosse redundante, sem desconsiderar questões de menor ocorrência. Para isso, foi-se utilizada uma abordagem Bottom-Up com o uso da técnica de diagrama de afinidades (Barnum, 2011; Garfney, 1999), adaptado às especificidades do estudo: 1) Primeiro, todas as descrições de tipos de erro foram coladas em fichas separadas, e depois agrupadas por similaridade de suas características; 2) o processo se repetiu, ajustando as fichas de lugar até alcançar um nível de similaridade satisfatório para toda a equipe de pesquisa; 3) as características de cada grupo foram analisadas e em seguida nomeadas de acordo com sua funcionalidade; 4) todas as fichas semelhantes foram agrupadas em apenas 1 tipo de erro e aquelas que se destoavam de todo o resto dos grupos receberam uma nomenclatura própria. 


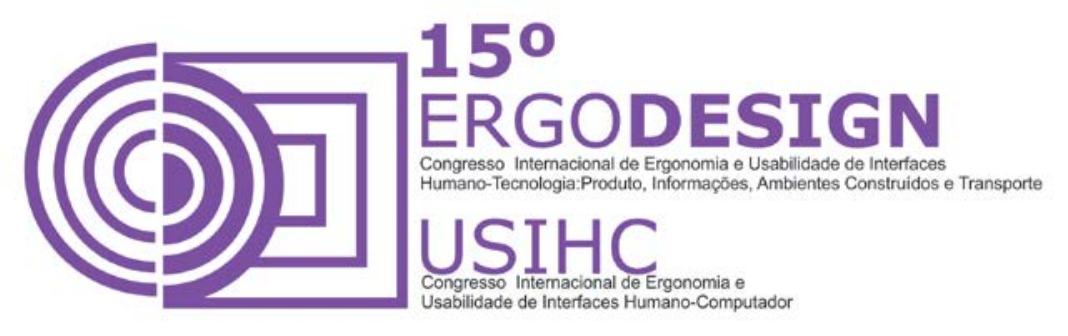

Ao fim da dinâmica, chegou-se ao que se acredita ser a lista dos possíveis tipos de erro humano no processo de interação com sistemas automatizados.

Para melhor entender como os diferentes tipos de erro ocorrem dentro do processo de interação, sentiu-se a necessidade de uma desconstrução das etapas que compõem cada um deles dentro da teoria que fundamenta o estudo da interação Humano-Automatização. Tal desconstrução permite um melhor entendimento das origens dos fenômenos listados, e como se aplicam em um cenário real- permitindo assim a melhor consideração dos erros durante 0 processo de concepção dos produtos. Para atender tal necessidade, foi utilizada uma modelagem dos tipos de erro dentro do esquema OODA LOOP(Thomas, 2001 apud Gikkas 2013). Como já dito anteriormente, para que a interação Humano-Automatização se dê de forma satisfatória, todos os processos descritos no OODA LOOP devem ser contemplados com a menor interferência possível, logo se caracteriza como erro, todo aquele estágio do esquema que não ocorre como o desejado de acordo com o modelo.

O processo da modelagem se deu da seguinte maneira: 1) Primeiramente foi definida uma interação virtualmente ideal de acordo com o esquema, servindo como base para a inserção do desvio causado pelo erro; 2) a partir da descrição do erro, o mesmo foi inserido dentro de um dos quatro estágios do loop (Observação, Orientação, Decisão e Ação); 3) uma vez devidamente inserido no processo, foi feita uma análise de quais dos três pilares (consciência da situação, confiança e carda de trabalho) da interação era comprometido por este tipo de erro; 4) caso fosse constatado que o pilar prejudicado pela ocorrência do erro fosse necessário para a interação, o esquema era redesenhado de acordo com a mudança, indicando como seria o processo caso tal erro ocorresse; 5) caso o pilar prejudicado em questão não fosse relevante para o estágio da interação em que o tipo de erro estivesse inserido, o mesmo era retirado e remanejado de acordo com uma nova análise; 6) uma vez encontrado o momento de sua ocorrência e a sua interferência na interação, o são buscadas possíveis causas para sua ocorrência, para que melhor sejam reconhecidas durante a sua aplicação em uma simulação de cenários.

\section{RESULTADOS}

Nesta etapa do artigo estão representados os tipos de erro encontrados no estudo. Primeiramente será exibido o modelo ideal de interação, onde todos os estágios do processo estão considerados de forma satisfatória, em seguida serão exibidos os erros descritos:

Modelo ideal: Para que não haja problemas durante a interação Humano-Automatização, fazse necessário que todos os 4 estágios do esquema OODA LOOP sejam contemplados de acordo com as necessidades do usuário, ou seja:1) Ele deve estar atento e ser capaz de observar com clareza todos os pontos referentes ao funcionamento do sistema; 2) ele deve ser capaz de distinguir e interpretar a informação observada de maneira a entender o que está se passando; 3) uma vez consciente da situação, deve ser capaz de tomar a decisão correta de acordo com o cenário; 4) ele deve ser capaz de executar a ação planejada sem grandes dificuldades e em tempo hábil. 


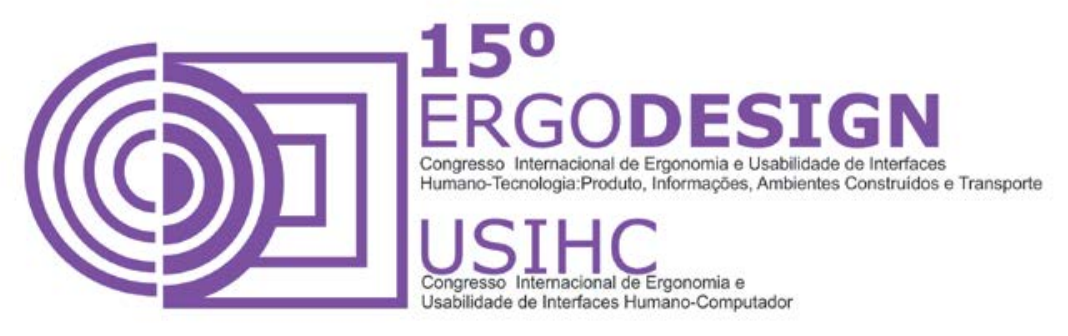

Erro de modo: Tipo de erro que ocorre quando o operador não tem a real noção do estado atual do sistema automatizado, fazendo com que o mesmo não consiga agir adequadamente durante uma situação que necessite a interferência direta no funcionamento do sistema. Muito comum em operadores com pouca familiaridade com o sistema operado, este tipo de erro ocorre no processo de orientação do usuário, e interfere diretamente em questões relacionados à consciência da situação.

Out of the Loop: Tipo de erro que ocorre quando a vigilância do usuário sob de determinada questão se encontra em níveis baixos ou inexistentes, não atentando para o funcionamento do sistema, logo, tornando-se incapaz de agir com destreza caso necessário durante uma situação anormal. Este tipo de erro ocorre no processo de observação por parte do usuário, comprometendo diretamente questões relacionadas à consciência da situação e carga de trabalho.

Excesso de confiança: Tipo de erro que ocorre quando apesar de observar uma anormalidade no funcionamento do sistema, o operador se recusa a aceitar tal questão como erro, confiando mais na precisão do sistema do que em si mesmo. Muito comum em operadores com pouca experiência com automatização, este tipo de erro ocorre no processo de decisão do usuário, e interfere claramente em questões relacionadas à consciência da situação e confiança do usuário.

Falta de confiança: Similar ao tipo de erro acima, este ocorre quando apesar de observar claramente o funcionamento normal do sistema automatizado, o usuário se nega a confiar nas ações realizadas pela máquina, interferindo desnecessariamente ou exercendo esforço demasiado em sua vigilância. Este tipo de erro ocorre no processo de decisão do usuário e interfere em questões de carga de trabalho e confiança

Vacilo de atenção: Tipo de erro diretamente ligado a questões de falibilidade humana - fadiga, distração e afins. Ocorre quando, apesar do estado de alerta, o operador mostra-se incapaz de observar todas as variáveis relevantes durante o funcionamento do sistema. Este tipo de erro ocorre no processo de observação do usuário e interfere em questões diretamente relacionadas à carga de trabalho.

Incapacidade operacional: Tipo de erro que ocorre quando, apesar da leitura correta do cenário e tomada de decisão precisa, o operador é incapaz de realizar as ações planejadas em tempo hábil, seja pela complexidade da tarefa ou pelo curto espaço de tempo. Este tipo de erro ocorre no processo de ação do usuário, interferindo em questões relacionadas exclusivamente à carga de trabalho.

Imprevisibilidade: Tipo de erro que ocorre quando o operador se mostra incapaz de determinar o modelo de funcionamento da máquina, fazendo com que o mesmo não consiga prever as reações por parte do sistema perante determinada situação eminente. Este tipo de erro ocorre no processo de orientação em conjunto com o processo de decisão - muito comum em operadores com pouca experiência com o sistema operado, interfere diretamente em questões relacionadas à consciência da situação. 


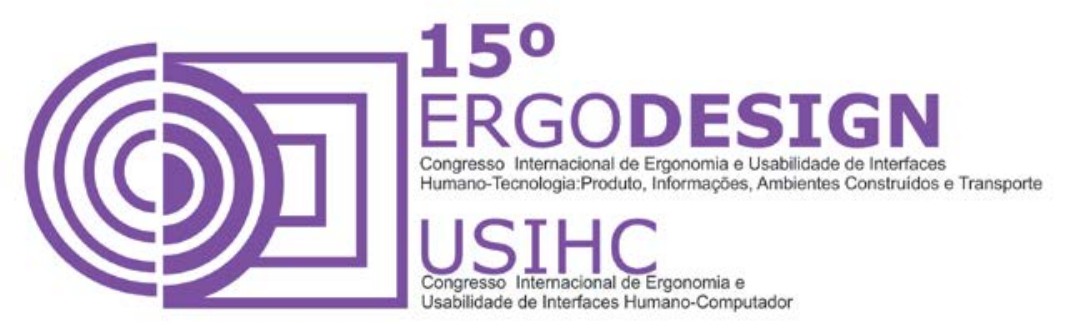

Erro de máquina: Tipo de erro que ocorre quando, apesar de uma interação perfeita entre humano e o sistema automatizado, ocorre uma falha de funcionamento da máquina, gerando resultados indesejados e imprevisíveis. Este tipo de erro ocorre durante o processo de execução da ação por parte da máquina, e não interfere diretamente em nenhuma questão relacionada ao usuário, uma vez que este tipo de falha não pode ser evitada pelo mesmo durante a operação.

Desconsideração intencional do sistema: Erro mais relacionado à prudência do usuário do que à interação em si, ocorre quando o operador intencionalmente desliga algum sistema relacionado à segurança e age de forma autônoma durante a execução de uma tarefa. Tal erro não é relacionado a nenhum dos processos comumente envolvidos na interação HumanoAutomatização - justamente por desconsidera-la, interfere diretamente em questões relacionadas à confiança.

\section{CONCLUSÃO}

Ao longo desta pesquisa foi realizada uma extensa revisão bibliográfica sobre interação Humano-Automatização o que levou a uma listagem dos mais prováveis tipos de erro que podem acontecer durante este tipo de interação. Tais erros foram catalogados e classificados a partir de um diagrama de afinidades e em seguida foram modelados dentro do esquema OODA para serem melhor analisados dentro de diferentes cenários de ocorrência.

O estudo constatou que cada um dos tipos de erro pode ser ocasionado por diferentes causas, que podem vir a se expressar de diversas formas em diferentes cenários. Normam (2013) afirma que razões para a ocorrência de erros são inúmeras. Algumas pela limitação da tecnologia. Outras por restrições feitas pelo design do produto, mas a maioria delas ocorre pela falta de conhecimento dos princípios que fundamentam a interação Humano-Máquina. Norman (2006) ainda afirma que devido à falta de terreno comum de comunicação entre humanos e máquinas, ainda não existem meios para que as mesmas considerem $100 \%$ dos possíveis causadores de erros durante a sua concepção. Levando em consideração a premissa acima e o fato do sistema automatizado ser estático (incapaz de aprender com os próprios erros de maneira precisa), ele deve ser capaz de lidar com todos estes tipos de erro uma vez que eles ocorram. Como não se pode prever o quando e o como eles podem vir a ocorrer devido à virtualmente infinita quantidade de cenários possíveis para a ocorrência de um erro, deve se considerar suas consequências e características para promover formas do usuário lidar com as falhas da melhor maneira possível. Com isso faz-se possível mitigar a gravidade dos problemas de interação e possivelmente reduzir a ocorrência de acidentes.

Acredita-se também que questões relacionadas a fatores sócio-culturais são de grande influência durante o a interação com sistemas automatizados (Parazuraman \& Sinderman, 2011; DEGANI, 2003; Thomas apud Gikkas, 2013). Com isso conclui-se que a adaptação do funcionamento do sistema a questões comportamentais do seu publico alvo - e não apenas a questões relacionadas aos diferentes cenários de ação do sistema, podem contribuir para a redução de ocorrência e/ou intensidade de diferentes tipos de erro - principalmente ligados à consciência da situação ou confiança. 


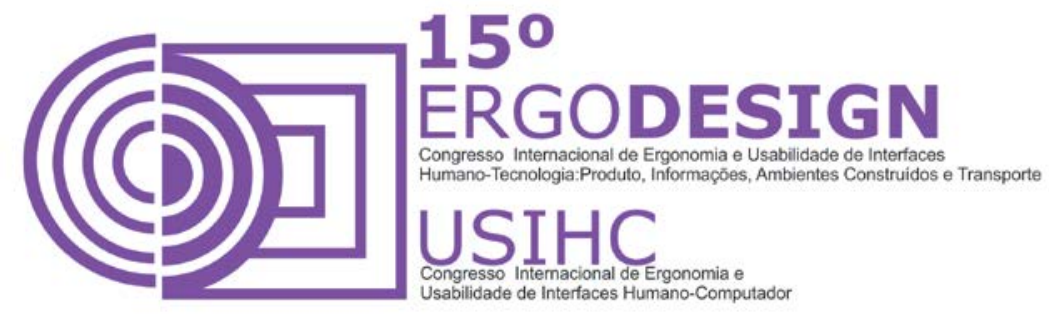

Para os desdobramentos da pesquisa pretende-se validar as hipóteses levantadas a partir de modelagens conceituais através de testes com simuladores e entrevistas com usuários. Uma vez validados, tais achados poderão ser aplicados na forma de diretrizes de concepção de sistemas automatizados tendo em vista a boa interação com os usuários.

\section{REFERÊNCIAS BIBLIOGÁFICAS}

BARNUM, C. Usability Test Essentials: Ready, Set... Test. 1 Edição, USA, Morgan Kufmann, 2010

CHAPANIS, A. Research Techniques in Engineering. Segunda Edição. Jhons Hopkins Press. Baltimore - Maryland. 1962.

DEGANI,; A. Taming Hal: Designing Interfaces Beyond 2001. Palgrave MacMillan. New York. 2003.

DEKKER, S. Ten Questions About Human Error: a new view of human factors and system safety. MAHWAH, 1997.

GARFNEY, G. Usability Techniques Series,<WWW.INFORMATIONDESIGN.AU>, USA, 1999.

GIKKAS ET AL. Automotive Ergonomics: Driver-Vehicle Interaction. CRC Press. UK. 2013.

IEC 61508-5. Functional Safety of Electrical/Electronic/Programmable Electronic Safety-Related Systems. 2009.

INTERNATIONAL STANDARIZATION ORGANISATION. ISO 26262. Road Vehicles and Functional Safety. 2011

KLETZ, T. An Engineer's View of Human Error. Taylor \& Francis. New York - NY. 2013

KOWALSKI, E. Erro Humano e Consciência da Situação. Ergodesign/USIHC 2013 (Congresso). Juiz de fora - MG. 2013

MORAES, A; MONT'ALVÃO, C. Ergonomia: Conceitos e aplicações. 4 edição. Rio de Janeiro. 2AB. 2012.

NORMAN, D. The Design of Future Things. Basic Books, 2009. 240 ISBN 0465002285.

PRARAZURAMAN, R. \& SINDERMAN. Human-Automation Interaction. HFES. USA. 2006.

PARASURAMAN, R; RILEY, V. Humans and Automation: Use, misuse, disuse abuse. HFES. 1997.

PROCTOR; VU. Task Analisys. In: SALVENDI, G. Handbook of Human Factors and Ergonomics. New Jersey. Willey. 2006.

REASON, J. Human Error. Cambridge University Press. New York - NY. 1990

SHARIT, J. Human Error. In: SALVENDI, G. Handbook Of Human Factors And Ergonomics. New Jersey. Willey. 2006.

YOUG, M, S; BRUCE, D. Driving Into The Sunset: Suporting Cognitive Function In Older Drivers. Journal Of Aging Research. 2011 